\title{
A novel hall thruster utilizing space debris as the propellant
}

\author{
Peng $\mathrm{E}^{1, \mathrm{a}}$ and Wenbin Ling ${ }^{2}$ \\ Harbin Institute of Technology, School of Electrical Engineering \& Automation, Harbin, China
}

\begin{abstract}
The paper proposed a new conception of using space debris as the working medium of the hall thruster. At first, we described differences of the physical properties between the space debris powder and gaseous propellants and estimated the charge quantity of the charged space debris powder. Then, we dealt with the operating principles and design process of the novel hall thruster with a modified cusped configuration of magnetic field along the discharge channel to make the ionization process more uniform and efficient. Given a design case, we demonstrated the validity of the novel idea through the simulation by the ANSYS Maxwell of the magnetic field distribution. The results are that the cusped configuration is 230 310 Gauss (increasing from the central line of discharge channel to the wall) in magnetic field and its gradient is 107 Gauss/cm, the maximum radial magnetic field is located in the exit plane of the thruster and has a 230 Gauss/cm gradient and a trend of inwards concave. This novel hall thruster with space debris as the propellant will not only augment the effective payload of the spacecraft and decrease the economic burden of the xenon propellant, but also it can resolve the space debris problem substantially.
\end{abstract}

Keywords: hall thruster; propellan; space debris; magnetic field; cusped configuration.

\section{Introduction}

Hall thruster has been a mature electric propulsion technology applied on the satellite and spacecraft to reduce the amount of the propellant required for a given space mission and application compared to other conventional methods. E.g, the applications include the attitude control, station-keeping of the geosynchronous communication satellites, orbit insertion, deep-space scientific exploration with the advantages of the appropriate specific impulse range, higher thruster density than ion thrusters, and the simpler structure as well as fewer power supply for its operation. And because of its merits of the xenon in several points, such as large mass for higher thrust, easily stored at high densities, and not hazardous to handle and process etc., the xenon is extensively used as the propellant at present [1-3].

However, the spacecraft takes the propellant as a part of its payload from the ground, so a large number of energy requirements would reduce the effective payload and affect the executive capability of the mission. Especially as to the long-term serving spacecraft, for example the ISS or the spacecraft for the deep-space exploration, it is more obvious that if the fuel is limited, the mission will be weakened. At the same time, the xenon is expensive and will increase the economic burden. Thus, if we could use local materials around the space environment of the orbit, not only the effective payload can be augmented but also it will give rise to great cost decreasing and the increasing of the on-orbit service time. It is known that the local materials defunct in the space environment are mainly the

\footnotetext{
${ }^{a}$ Corresponding author : epeng@hit.edu.cn
} 
space debris, whose number has been rising up with the conducting of the human space activities, leading to great threat to the space exploration and applications which play a considerable role in modern society.

According to the IADC (Inter-Agency Space Debris Coordination Committee), orbital debris is defined as any man-made object in orbit which no longer serves a useful purpose and space debris includes natural meteoroids as well [4]. Currently, there are about 22000 objects whose size are $10 \mathrm{~cm}$ or bigger tracked by the AFSSS (Air Force Space Surveillance Systems), 500000 objects with size bigger than $1 \mathrm{~cm}$ and many millions of objects smaller than $1 \mathrm{~mm}$ in size on orbit and the number still grows rapidly because of their self-propagating. The damage potential of the spacecraft due to the collision with the space debris is rather large, the hypervelocity motion of space debris at a speed of or so $7 \mathrm{~km} / \mathrm{s}$ implying tremendous kinetic energy. That no spacecraft can defend this risk gives rise to concerns that the potential problem must be overcome so that the spacecraft operates safely and reliably [5].

To address this threat, people have used a lot of relevant methods in terms of the types of the space debris, from the spacecraft maneuver and avoidance for large debris to the shielding project of the spacecraft's structures and materials. In addition to these passive cases, people also pursue to improve the status through mitigation guidelines that can alleviate it temporarily or active removal technologies that can resolve it permanently. Even so, the situation is not positive when the complexity of the matter and the deterioration of the present space environment are confronted, especially the incident of the anti-satellite test in 2007 and the explosion of satellites in 2009 occur. All those methods existing would involve multi-field cooperation such as politics, regulation and legislation, economy, let alone the multidisciplinary technology that includes the modeling of the space debris orbital evolution, detecting and tracking the space debris using radar or optical systems, as well as extensive constraints related to the specific cases. From the perspective of the long-term sustainability of outer space, the active space debris capturing and removal methods are the most promising ways that will function well despite the long way to go.

There are many capturing and removal methods existing in conception, but they all just treat the space debris as hazard that must be addressed without the consideration of utilizing it for other unexpected effects [6, 7]. As described previously, utilizing space debris addressed as the propellant for the hall thruster will be a good way of using the local materials to substitute the xenon. So, the facts about the hall thruster and the space debris status directs us to use the space debris as the propellant, which will settle the space debris problem effectively. Similar to this idea, there are some literature suggests adopting the atmospheric air as the propellant $[8,9,10]$. In addition, Lei Lan et al. [11] has proposed a kind of debris engine that using the space debris as the propellant transferred from the space robotic cleaner, disintegrated into the power and charged by the gamma-ray photoelectric effect or the behavior of tangent rub of tungsten needles, which would be accelerated by a electrostatic particle accelerator ejected in the form of high-temperature and high-pressure charged powder providing the thrust. That is a good idea suggesting the utilization of space debris, but it has some drawbacks such as the low thrust density and the serious erosion of the gridding electrodes that determines the efficiency of the thruster, which can be overcome by the hall thruster through founding the matching performance between the space debris powder and corresponding thruster to find the optimal operational environment and conditions. So, a novel hall thruster utilizing space debris as the propellant would be a creative and reasonable way of treating the space debris and offering the power for the spacecraft.

This paper proposes the new idea of utilizing space debris as the propellant for the hall thruster, which demands the preceding capturing and smashing operations supplying the necessary space debris powder with proper properties matching the conditions of the thruster. Here, we just depict the parameters of desired space debris powder not to elaborate the specific process for the space debris, but closely concentrate on the design of the structure and analyze the operating conditions of the hall thruster to match with the particular propellant. Thus, the paper is structured as follows. Lying in the introduction section is the background of the new idea. Then in the second part, we show the distinction between the space debris powder and gaseous propellants. The structure and operational 
principles of the hall thruster with the space debris as the propellant are analyzed and demonstrated in detail in the third part. At the same time, after these theoretical aspects, this section also accounts for the design specifications of the novel hall thruster and simulation results. In the last, the conclusion part summarizes the paper and evaluates the new idea here, while it also indicates the deficiency that should be overcome in the future work.

\section{The distinction with respect to gaseous propellants}

Belonging to the realm of dusty plasmas, the physical characteristics of the propellant of space debris powder is largely different from those of gaseous propellants in various aspects, which is critical in designing the corresponding hall thruster to face the new plasma environment. First of all, the most obvious difference is that the size of the space debris powder is quite a few orders of magnitude more than the gaseous propellants so that the space debris powder appears as the solid-state and with bigger mass directly resulting in the harder problem of the diffusion than the gas based on the effect of the weight. Therefore, the issue would influence the homogeneity of the propellant as well as the mechanism of the ionization and thus the efficiency of the thruster, luckily however, this trouble can be eliminated in the weightless space and only need to be considered in the ground test. The second difference lies in the ionization process of the space debris on account of the cross section several magnitudes bigger than that of xenon and there exits the considerable combination process when the space debris possesses a number of positive charges, Leading to a result of the balance must be considered. Due to the process is rather intricate, the paper just do a hypothetical handle as the later paragraph shows. With the distribution of the pulverous particles mass and the complexity of the ionization, it is the third difference adverse to stability and continuity of the thruster that the degree of dispersion of the charge-to-mass ratio about the space debris powder is large. As will be seen later, this situation can be compensated by applying the cusped configuration of the magnetic field along with the discharge channel which decreases the wall erosion and integrates the ionization and acceleration regions making the thruster more compact.

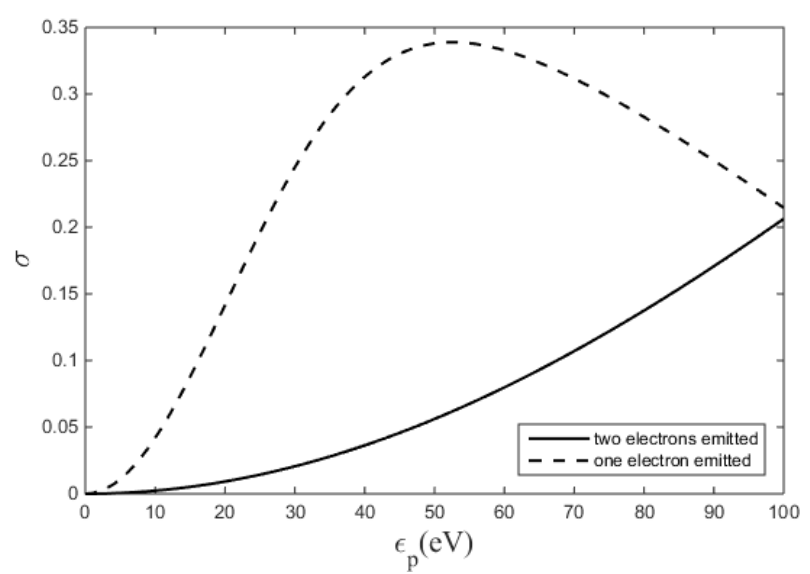

Figure 1. The probability of the emitted electrons.

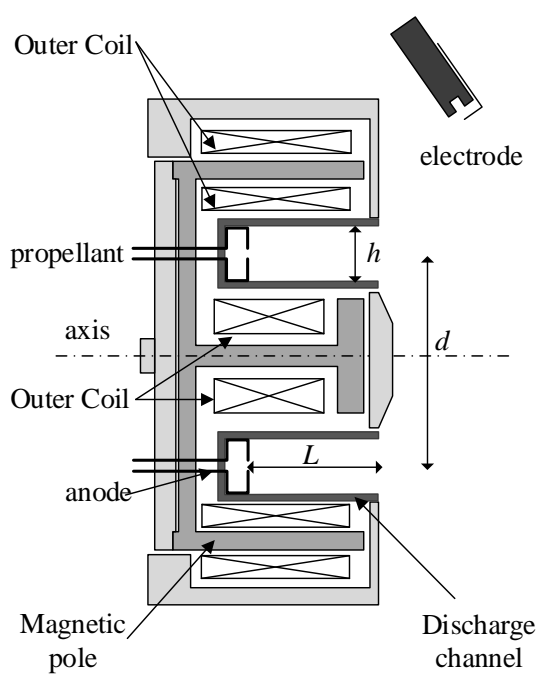

Figure 2. The structure of the thruster.

Now, we consider an electron beam coming from the electrode with a macroscopic kinematic velocity $v$, a thermal velocity with energy presentation $\varepsilon_{\mathrm{p}}$ whose unit is $\mathrm{eV}$. The density of the beam is $n_{\mathrm{e}}$ unit in $\mathrm{m}^{-3}$, the radius of the space debris is $r$ and the time of charge is $t$. Consider the model of the secondary emission of the space debris, we can describe the probability of the electrons accumulated on the space debris powder, the electrons collided elastically, two electrons emitted and one electron as $W_{0}\left(\varepsilon_{\mathrm{p}}\right), W_{\mathrm{r}}\left(\varepsilon_{\mathrm{p}}\right), W_{2}\left(\varepsilon_{\mathrm{p}}\right)$ and $W_{1}\left(\varepsilon_{\mathrm{p}}\right)$ respectively [12] 


$$
\begin{gathered}
W_{0}\left(\varepsilon_{\mathrm{p}}\right)=0.85 \exp \left(-\varepsilon_{\mathrm{p}}^{2} / 2422^{2}\right) \\
W_{r}\left(\varepsilon_{\mathrm{p}}\right)=0.42 \exp \left(-\varepsilon_{\mathrm{p}}^{2} / 30^{2}\right) \\
W_{2}\left(\varepsilon_{\mathrm{p}}\right)=1-\exp \left(-\varepsilon_{\mathrm{p}}^{2} / 208^{2}\right) \\
W_{1}\left(\varepsilon_{\mathrm{p}}\right)=1-W_{0}\left(\varepsilon_{\mathrm{p}}\right)-W_{r}\left(\varepsilon_{\mathrm{p}}\right)-W_{2}\left(\varepsilon_{\mathrm{p}}\right) \\
q_{\mathrm{i}}=\pi r^{2} v n_{e} t\left[W_{1}\left(\varepsilon_{\mathrm{p}}\right)+W_{2}\left(\varepsilon_{\mathrm{p}}\right)\right]
\end{gathered}
$$

The Figure 1 plot the $W_{2}\left(\varepsilon_{\mathrm{p}}\right)$ and $W_{1}\left(\varepsilon_{\mathrm{p}}\right)$ relative to the $\varepsilon_{\mathrm{p}}$ to illustrate the positive charge of the space debris powder. We can obtain the charge quantity $q_{\mathrm{i}}$ of the charged space debris powder as (5) when the adsorption of the electrons is ignored. Despite of its failure when the balance of the charge current is considered, it still is a rough estimation of the charge quantity.

\section{The operating principles and design of the thruster}

As the Figure 2 shows, the hall thruster comprises the cathode and anode which provide the potential drop, the inner and outer coils plus the magnetic poles by which the almost radial magnetic field is formed around the circular discharge channel, as well as the anode propellant inlet tube. The basic process of the hall thruster is as follows. When the thruster is activated, the high-energy electrons are emitted from the electrode and attracted to the anode under the axial electric field, but they will be confined in the discharge channel because of the magnetic field with a few conducting towards the anode. So the high-energy electrons are subjected to the cross field of $\boldsymbol{E}$ and $\boldsymbol{B}$, drifting in the circumferential direction to generate the current of this closed drift. The space debris powder injected through the inlet tube is travelling towards the cathode, colliding with the high-energy electrons in the ionization region of the channel to turn into the ions which will be speeded up in the acceleration region and ejected from the nozzle of the hall thruster as the so-called plume. The highspeed ions containing large momentum provide the thrust and then are neutralized by the electrons to sustain the electric neutrality of the entire system [13-16]. The operating principles which maintain the engineering design of the thruster are indicated by the inner physical nature of the thruster and we will discuss them in detail in the following paper.

In order to guarantee the effective ionization of the space debris powder, the length of the discharge channel must conform to the Melikov-Morozov criterion, which is a kind of similarity rules, claiming the mean free path $\lambda_{\mathrm{i}}$ of the ionization process is much smaller the length of the discharge channel $L$

$$
\lambda_{\mathrm{i}}=\frac{v_{\mathrm{n}}\left(T_{\mathrm{n}}\right)}{n_{\mathrm{n}} \sigma_{\mathrm{i}}\left(T_{\mathrm{e}}\right) v_{\mathrm{e}}\left(T_{\mathrm{e}}\right)}<<L
$$

$v_{\mathrm{n}}\left(T_{\mathrm{n}}\right)$ is the thermal velocity of the space debris, unit in $\mathrm{K} ; n_{\mathrm{n}}$ is the density of the space debris powder, unit in $\mathrm{kg} \cdot \mathrm{m}^{-3} ; \sigma_{\mathrm{i}}\left(T_{\mathrm{e}}\right)$ is the collision cross-section of the ionization process, unit in $\mathrm{m}^{2} ; v_{\mathrm{e}}\left(T_{\mathrm{e}}\right)$ is the the thermal velocity of the high-energy electrons, unit in $\mathrm{K}$.

In order to insure the high-energy electrons are confined in the magnetic field and the highvelocity ions can be suitably ejected from the hall thruster exit, the width of the discharge channel must satisfy the relation of $r_{\mathrm{ce}}<<h<<r_{\mathrm{ci}}$, where the $r_{\mathrm{ce}}$ and $r_{\mathrm{ci}}$ stand the cyclotron radius of the electrons and ions located in the magnetic field 


$$
\begin{gathered}
r_{\text {ce }}=\frac{m_{\mathrm{e}} v_{\mathrm{e}}\left(T_{\mathrm{e}}\right)}{\mathrm{e} B} \\
r_{\mathrm{ci}}=\frac{m_{\mathrm{i}} v_{\mathrm{i}}\left(T_{\mathrm{i}}\right)}{q_{\mathrm{i}} B}
\end{gathered}
$$

Wherein, the e denotes the essential charge of the electron, unit in $\mathrm{C} ; v_{\mathrm{i}}\left(\mathrm{T}_{\mathrm{i}}\right)$ is the thermal velocity of the charged space debris powder, unit in $\mathrm{K} ; m_{\mathrm{e}}$ is the mass of the basic charge of electron, unit in $\mathrm{kg} ; m_{\mathrm{i}}$ is the mass of the charged space debris, unit in $\mathrm{kg}$.

In order to increase the degree of the restraint of the electrons by the magnetic field and decrease the loss of the electrons traversing the magnetic field lines thus the conducting current of the thruster, the cyclotron frequency should be much bigger than the collision frequency between the electrons and the space debris powder

$$
\frac{\omega_{\text {ce }}}{v_{\text {en }}}=\frac{\mathrm{e} B}{m_{\mathrm{e}} n_{\mathrm{n}} \sigma_{\mathrm{en}} v_{\mathrm{en}}}>>1
$$

In this expression, $\omega_{\text {ce }}$ is the cyclotron frequency of the electrons, unit in $\mathrm{Hz} ; v_{\mathrm{en}}$ is the collision frequency between the electrons and the pace debris powder, unit in $\mathrm{Hz} ; \sigma_{\mathrm{en}}$ is the cross section of the exchange of the momentum, unit in $\mathrm{m}^{2}$.

The approach to determine the characteristic parameters of the thruster is based on that operating principles and also the practical or experienced rules. The procedure of identify the parameters include these steps that given the magnetic field $B$, discharge voltage $U_{\mathrm{d}}$ and the thruster $T$, decide the appropriate structural dimensions $L, h, d$ as well as the mass rate of the propellant $\dot{m}$ according to the operational conditions of the thruster coupled with the scaling laws related to the parameters and the properties ( the thruster and the specific impulse etc. ), when the empirical rules yielded from the database of the existing thrusters $[17,19]$. The steps is as follows

(1) Identify the mass rate of the propellant $\dot{m}$ on the basis of the relationship about the thruster $T$, the discharge voltage $U_{\mathrm{d}}$ and the $\dot{m}, C_{\mathrm{T} 1}=1.0773$

$$
T=C_{\mathrm{T} 1} \dot{m} \sqrt{U_{\mathrm{d}}}
$$

(2) The average diameter $d$ of the discharge channel can be acquired through the expression that describes the relation about the thruster $T$, the $d$ and the discharge voltage $U_{\mathrm{d}}, C_{\mathrm{T} 2}=0.613$

$$
T=C_{\mathrm{T} 2} \sqrt{U_{\mathrm{d}}} d^{2}
$$

(3) Determine the width of the discharge channel $h$ based on the aforementioned relation $r_{\mathrm{ce}}<<h$ $<<r_{\mathrm{ci}}$, then we can have the length of the discharge channel $L$ according to the empirical rule $L \sim 5 h$ and we should also examine the validity of the restrict $\lambda_{\mathrm{i}}<<L$.

(4) Amend the value of the $B$ in view of the condition of the effective magnetization of the electrons, namely $\omega_{\text {ce }} \gg v_{\text {en }}$.

(5) Choose the $B$ and $U_{\mathrm{d}}$ in another process and repeat the steps of calculation here to find the most optimal properties of the thruster.

Because the space debris powder is complex in the components and they have large differences in the ionization energy, there would be longer ionization region demanded for the effective ionization if the traditional thruster is used. In addition, the charged space debris is different in charge-to-mass ratio largely so that plasma plume is not continuous out of the thruster, thus this will give rise to the decline in the control accuracy, stability and efficiency. The essential reason for this is that the ionization and acceleration regions are separated resulting two functional sections in space. So, only 
the entirely ionized propellant will obtain the energy from the electric field and be ejected out of the thruster in velocity several ten times larger than neutral propellant, which suggests requirement for the uniformity for the space debris powder that cannot be satisfied in traditional thruster. In order to pursue the thruster design matching with the particular propellant, the paper proposes that applying the cusped configuration of the magnetic field will make the ionization more efficient due to the decrease of the collision of the space debris powder and electrons with the channel wall [20-25]. The cusped configuration is achieved through introducing the extra two coils and corresponding magnetic yokes based on the primary thruster as shown in Figure 3. Then, we will give a case showing the design of the hall thruster with the space debris powder as the propellant.

At First, we present the operating parameters requirements. The feature dimension of the space debris powder whose density is estimated using the Si is in submicron magnitude and the quantity of the basic charge is $10^{5}$ or so. The discharge voltage between anode and cathode is in the range of $200 \sim 1000 \mathrm{~V}$, and the radial magnetic field $B$ in the channel has the value of $0.02 \mathrm{~T} \sim 0.1 \mathrm{~T}$ with a positive gradient $200 \mathrm{Gauss} / \mathrm{cm}$ (experiments shows a good stability with such magnetic configuration) towards the exit of the thruster. The magnetic field of the cusped configuration $B_{1}$ has the value of $0.02 \mathrm{~T} \sim 0.1 \mathrm{~T}$, with a positive gradient pointing to the discharge channel radially. Suppose the energy presentation of the thermal velocity of the electrons is $10 \mathrm{eV}$, the initial velocity of the space debris is $v_{\mathrm{i} 0}\left(T_{\mathrm{i}}\right)=500 \mathrm{~m} / \mathrm{s}$, so

$$
\begin{gathered}
\frac{1}{2} m_{\mathrm{e}} v_{\mathrm{e}}^{2}\left(T_{\mathrm{e}}\right)=10 \mathrm{eV} \\
v_{\mathrm{i}}\left(T_{\mathrm{i}}\right)=v_{\mathrm{i} 0}\left(T_{\mathrm{i}}\right)+\left(2 q_{\mathrm{i}} U_{\mathrm{d}} / m_{\mathrm{i}}\right)^{1 / 2} \\
m_{\mathrm{i}}=\frac{4}{3} \pi r^{3} \rho_{\mathrm{Si}}
\end{gathered}
$$

$m_{\mathrm{i}}$ is the mass of the individual space debris powder, unit in $\mathrm{kg} ; \rho_{\mathrm{si}}=2.33 \times 10^{-3} \mathrm{~kg} / \mathrm{cm}^{3}$ stands for the density of the Si element. When $n_{\mathrm{e}}=10^{18} \mathrm{~m}^{-3}$, the $q_{\mathrm{i}}$ is in the $10^{5}$ order and here we set $q_{\mathrm{i}}=10^{5} \mathrm{C}$. Thus, If $U_{\mathrm{d}}=200 \mathrm{~V}, \quad r_{\mathrm{ce}}=0.11 \mathrm{~mm} \sim 0.53 \mathrm{~mm}, \quad r_{\mathrm{ci}}=7.99 \mathrm{~m} \sim 39.91 \mathrm{~m} ; \quad U_{\mathrm{d}}=1000 \mathrm{~V}, \quad r_{\mathrm{ce}}=0.11 \mathrm{~mm} \sim 0.53 \mathrm{~mm}$, $r_{\mathrm{ci}}=14.09 \mathrm{~m} \sim 70.42 \mathrm{~m}$. When the $B$ is larger than $0.02 \mathrm{~T}$, the $r_{\mathrm{ce}}$ will be smaller than $0.5 \mathrm{~mm}$, so we choose $h \sim 3 \sim 5 \mathrm{~cm}, L \sim 5 h=15 \mathrm{~cm} \sim 20 \mathrm{~cm}$; According to the previous design procedure, we can also acquire $d=51 \mathrm{~mm} \sim 76 \mathrm{~mm}, \dot{m}=1.47 \sim 3.29 \mathrm{mg} / \mathrm{s}$. In addition, we should consider the restrictions from the magnetic coils when determine the relative dimensional relation.

We can call the modified hall thruster with the cusped magnetic field configuration the integrated hall thruster of ionization and acceleration. The magnetic field quantity of this configuration is decided by the specific pressure which is the ratio of the pressure of the plasma and that of the magnetic field

$$
\beta=\frac{n_{\mathrm{i}} \mathrm{k} T_{\mathrm{i}}+n_{\mathrm{e}} \mathrm{k} T_{\mathrm{e}}}{B_{1}^{2} / 2 \mu_{0}} \ll 1
$$

$n_{\mathrm{i}}$ is the density of the space debris powder, unit in $\mathrm{m}^{-3}$; $\mathrm{k}$ stands for the Boltzmann constant, $\mathrm{k}=1.38 \times 10^{-23} \mathrm{~J} / \mathrm{K} ; T_{\mathrm{i}}$ is the temperature of the space debris powder, with its value $T_{\mathrm{i}}=300 \mathrm{~K} ; T_{\mathrm{e}}$ is the temperature of the electrons, $T_{\mathrm{e}}=1.16 \times 105 \mathrm{~K} ; \mu_{0}$ is the permeability of the free space, $\mu_{0}=4 \pi \times 10-7 \mathrm{H} / \mathrm{m}$; The density $n_{\mathrm{i}}$ can be calculated from the anode mass rate and the geometry of the thruster

$$
n_{\mathrm{i}}=\frac{\dot{m}}{v_{\mathrm{i} 0}\left(T_{\mathrm{i}}\right) m_{\mathrm{i}} \pi\left(r_{2}^{2}-r_{1}^{2}\right)}
$$




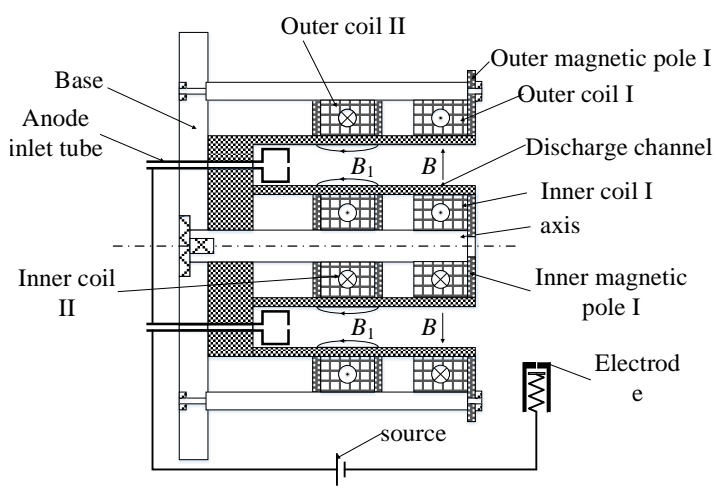

Figure 3. The modified hall thruster.

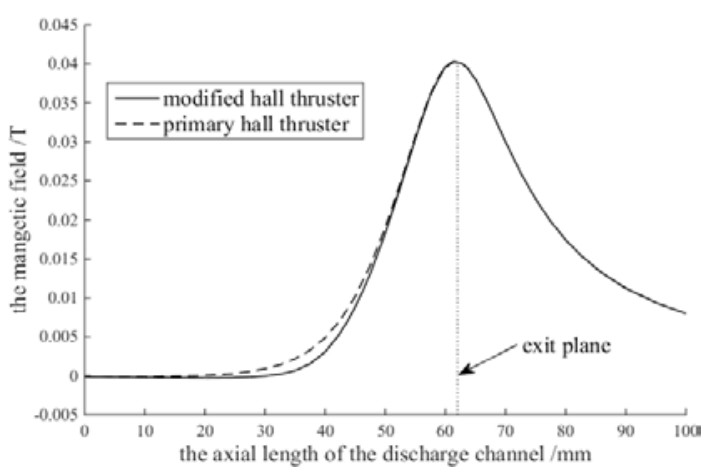

Figure 4. The axial distribution of the magnetic field B.

$r_{2}$ is the outer radius of the discharge channel, unit in $\mathrm{m} ; r_{1}$ is the inner radius of the discharge channel, unit in $\mathrm{m}$; When the $B_{1}$ equals $0.02 \mathrm{~T}$, the specific pressure $\beta$ is in $10^{-3}$ magnitude, which satisfies the design demand.

The left two in Figure 5 shows the simulated results of the magnetic field distribution in the modified hall thruster only without two extra coils compared with the primary hall thruster. The simulation is executed by the ANSYS Maxwell and the geometrical parameters are $h=15 \mathrm{~mm}$, $L=62 \mathrm{~mm}, d=51 \mathrm{~mm}$. The four coils all have the same cross section of $15 \mathrm{~mm} \times 10 \mathrm{~mm}$ and the same current of $500 \mathrm{~A}$ with a current density $3.33 \mathrm{~A} / \mathrm{mm}^{2}$ within the limit of the insulation temperature of the copper. The simulation results indicates that the hall thruster design can generate the required magnetic configuration. The cusped configuration is $230 \sim 310$ Gauss (increasing from the central line of discharge channel to the wall) in magnetic field and the gradient of this field is $107 \mathrm{Gass} / \mathrm{cm}$. As the figure depicted, there is a little difference of the magnetic field distribution in exit of the modified thruster compared with the primary thruster (Figure 5). The maximum radial magnetic field is located in the exit plane and there is a 230Gass/cm gradient shown in the Figure 4 . This inwards concave magnetic field configuration shows good stability of the hall thruster in the experiment.

\section{Conclusion and discussion}

This paper proposed a novel hall thruster utilizing space debris as the propellant by which the quantity of the working medium xenon can be decreased substantially and the effective payload is increased, and the new hall thruster can also relieve the threat of the space debris by making use of it creatively. In the background section, the paper deduced its objective after analyzing the status of present propellant utilization of xenon and connecting the hazard and capability of utilizing of the space debris. Based on its central goal, the paper listed its structural layout and internal logic relationships about each section.

The most obvious difference is the properties of the space debris powder compared with the xenon, so in the second section, the paper underlines the distinction from the physical characteristics that is key to the operating of the thruster including the size and material state of the space debris powder, the mechanism of the ionization and the charge-to-mass ratio of the ionized space debris powder. Upon these features, we design a novel hall thruster modified through adding a cusped configuration of the magnetic field along the discharge channel. The third section is the major part of the paper and treats the hall thruster operating and design in detail. 

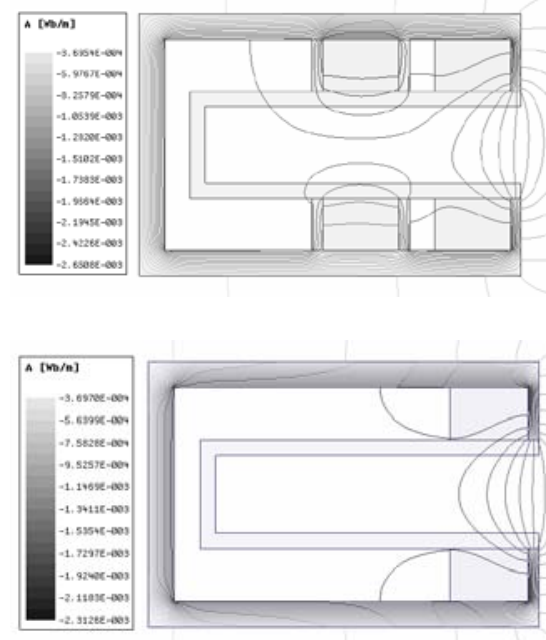

Figure 5. The magnetic field distribution of modified hall thruster (up) and primary hall thruster (down)

Firstly, the third section shows the basic physical picture and operating principles of the thruster from three aspects that are the Melikov-Morozov criterion, the relative relations about the width of the discharge channel and the cyclotron radius of the particles as well as the effective magnetization condition of the electrons. After the discourse, we unfold the design procedures of the parameters of the hall thruster according to the empirical rules and scaling laws. We designed a novel hall thruster whose characteristic operating requirements are set for space debris powder size and density, the charge quantity of it, the discharge voltage, the radial magnetic field and the cusped configuration of the magnetic field added. At last, we simulate the design project in the ANSYS Maxwell to validate its reasonability and the results meet with the desired very well. Especially, the cusped configuration of the magnetic field is achieved by the modified structure with the proper engineering limits very well. It lie in $230 \sim 310$ Gauss (increasing from the central line of discharge channel to the wall) in magnetic field and the gradient of this field is $107 \mathrm{Gass} / \mathrm{cm}$ ).

In brief, the new idea of utilizing the space debris as the propellant is reasonable in physics and viable when combing the available engineering experience of exiting hall thruster. Nevertheless, it is just a good conception which demands deep and extensive research about too many significant problems. The most important issue is that the ionization process of the space debris powder must be examined theoretically and experimentally to completely understand the physical nature of the space debris powder when exposed in the complex plasma environment. Then, we should provide new scaling laws and design considerations by surveying the dynamic characteristics of the charged space debris and the high-energy electrons using the complex physical models for example the kinetic or the fluid description of them in the discharge channel. In addition, the plume stability must be pay attention to acquire the control accuracy and optimal properties of the novel hall thruster, which should be investigated in the laboratory. At the same time, the specific conducting patterns of the electrons and the anode mass rate should be studied in order to improve the efficiency, reliability and lifetime of the novel hall thruster. Finally, there will need experimental set up to verfiy the novel conception of the improved hall thruster based on avaliable facilities of the plasma propulsion test.

\section{References}

1. MG. Dan, I. Katz. Fundamentals of Electric Propulsion (Wiley \& Sons, Inc., Hoboken, New Jersey, 2008).

2. B. B. Kadomtsev, VD. Shafranov. Reviews of plasma physics 21 (Kluwer Academic, New York, 2000).

3. C. Charles. J. Phys. D: Appl. Phys, 42, 163001-163018 (2009). 
4. JN. Pelton. New solutions for the space debris problems (Springer, Heidelberg, New York, Dordrecht, London, 2015).

5. D. Wright. PHYS TODAY, 60, 35-40 (2007)

6. M. Shan, J. Guo, E. Gill. PROG AEROSP SCI, 80, 18-32 (2015).

7. M. Kaplan. AIAA Space Conference \& Exposition (2009).

8. A. Greig, CH. Birzer, M. Arjomandi. AIAA J, 51, 362-371 (2013).

9. L. Garrigues. J PROPUL POWER, 28, 344-354 (2012).

10. L. Pekker, M. Keidar. J PROPUL POWER, 28, 1399-1405 (2015).

11. L. Lan, J. Li, H. Baoyin. Physics, (2015).

12. D. Ping, Q. Hai-Juan, Z. Xin-wei etc. Acta Phys. Sin., 63, 286-292 (2014)

13. VV. Zhurin, PLASMA SOURCES SCI T, 8, R1-R20 (1999).

14. JC. Adam, JP. Boeuf, N. Dubuit etc. PLASMA PHYS CONTR F, 50, 729-747 (2008).

15. Lyon B. King. The 29th International Electric Propulsion Conference, (2005).

16. M. Keidar, II. Beilis. IEEE T PLASMA SCI, 34, 804-814 (2006).

17. K. Dannenmayer, S. Mazouffre. J PROPUL POWER, 27, 236-245 (2011).

18. AA. Shagayda, OA. Gorshkov. J PROPUL POWER, 29, 466-474 (2013).

19. AA. Shagayda. IEEE T PLASMA SCI, 43, 12-28, (2015).

20. S. Mazouffre, G. Bourgeois, J. Vaudolon, etc. J PROPUL POWER, 31, 1-8 (2015).

21. NB. Meezan, HW. Jr, MA. Cappelli. PHYS REV E, 63, 235-241 (2001).

22. E. Ahedo, D. Escobar. J. APPL PHYS, 96, 983-992 (2004).

23. K. Dannenmayer, S. Mazouffre. Physics, 49, 231-254 (2008).

24. L. Garrigues, GJM. Hagelaar, J. Bareilles etc. PHYS PLASMAS, 10, 4886-4892 (2003).

25. P. Phariseau. PHYS PLASMAS, 8, 3058-3068 (2001). 